\title{
Insect endosymbiont proliferation is limited by lipid availability
}

\author{
Jeremy K Herren ${ }^{1 *}$, Juan C Paredes¹, Fanny Schüpfer ${ }^{1}$, Karim Arafah², \\ Philippe Bulet ${ }^{2,3}$, Bruno Lemaitre ${ }^{1 *}$
}

${ }^{1}$ Global Health Institute, School of Life Sciences, Ecole Polytechnique Fédérale de Lausanne (EPFL), Lausanne, Switzerland; ${ }^{2}$ Platform BioPark Archamps, Saint Julien en Genevois, France; ${ }^{3}$ Université Joseph Fourier, AGIM FRE CNRS, La Tronche, France

\begin{abstract}
Spiroplasma poulsonii is a maternally transmitted bacterial endosymbiont that is naturally associated with Drosophila melanogaster. S. poulsonii resides extracellularly in the hemolymph, where it must acquire metabolites to sustain proliferation. In this study, we find that Spiroplasma proliferation specifically depletes host hemolymph diacylglyceride, the major lipid class transported by the lipoprotein, Lpp. RNAi-mediated knockdown of Lpp expression, which reduces the amount of circulating lipids, inhibits Spiroplasma proliferation demonstrating that bacterial proliferation requires hemolymph-lipids. Altogether, our study shows that an insect endosymbiont acquires specific lipidic metabolites from the transport lipoproteins in the hemolymph of its host. In addition, we show that the proliferation of this endosymbiont is limited by the availability of hemolymph lipids. This feature could limit endosymbiont over-proliferation under conditions of host nutrient limitation as lipid availability is strongly influenced by the nutritional state.
\end{abstract}

DOI: 10.7554/eLife.02964.001

*For correspondence: jeremyherren@me.com (JKH); bruno.lemaitre@epfl.ch (BL)

Competing interests: The authors declare that no competing interests exist.

Funding: See page 17

Received: 30 March 2014 Accepted: 14 July 2014 Published: 15 July 2014

Reviewing editor: Utpal Banerjee, University of California, Los Angeles, United States

(c) Copyright Herren et al. This article is distributed under the terms of the Creative Commons Attribution License, which permits unrestricted use and redistribution provided that the original author and source are credited.

\section{Introduction}

Many insects harbor facultative bacterial endosymbionts, which despite not being required for host survival have important implications for host biology (Wernegreen, 2012). Two of the most prevalent and well-characterized facultative insect endosymbionts are Wolbachia and Spiroplasma, which are estimated to infect $\sim 40 \%$ and $5-10 \%$ of all insects species, respectively (Hackett and Clark, 1979; Duron et al., 2008; Hilgenboecker et al., 2008). While Wolbachia principally resides intracellularly (Dobson et al., 1999; Albertson et al., 2009), Spiroplasma occupies an extracellular niche, proliferating mainly in the hemolymph that fills the body cavity of arthropods (Sakaguchi and Poulson, 1961; Anbutsu and Fukatsu, 2006). Spiroplasma and Wolbachia are both maternally transmitted and have developed unique strategies to colonize the germline of their female hosts for transmission to the next generation (Frydman et al., 2006; Serbus and Sullivan, 2007; Herren et al., 2013).

Facultative endosymbionts with strict maternal transmission, including Wolbachia and Spiroplasma, increase their prevalence in host populations by virtue of two strategies: (i) manipulating host reproduction to increase the fitness of infected hosts (Werren and O'Neill, 1997); (ii) inducing a direct increase in host fitness in a manner that is usually condition dependent, for example protecting hosts against different classes of parasites (Hedges et al., 2008; Jaenike et al., 2010; Teixeira et al., 2008). Protective endosymbionts of disease vectors may be useful for the control of vector borne disease, and they are increasingly being studied in this context (Moreira et al., 2009). While these interactions are clearly of importance, more fundamental features of facultative endosymbioses are poorly understood and frequently overlooked, including metabolic exchanges and the mitigation of host fitness costs.

Genome sequencing has indicated that endosymbiotic bacteria have highly reduced metabolic capacities and depend heavily on their hosts to provide them with a diversity of compounds needed for their sustained proliferation (Klein et al., 2012; Moran et al., 2008). However, the direct study of 
eLife digest All animals host a large number of harmless microbes. Often the two partners involved in these interactions will depend on each other to thrive: microbes support important host functions and in return the host provides a safe place to live and a continuous supply of food. Many microbes that are intimately associated with animals have lost the ability to gain nutrients from sources other than their host and are unable to survive on their own. However, in many cases, the source and the type of nutrients provided to the microbes are unknown.

One of the most common microbial species found in insects is Spiroplasma. This microbe lives in very large numbers in the fluid that fills the body cavities of insects, called the hemolymph. The microbes are transmitted from mother to offspring, and in some circumstances can provide benefits to the insects; for instance, Spiroplasma-infested flies appear to be protected against infection by some parasites. Unfortunately, as it is difficult to study insect-microbe relationships, little else is known about the physiological interactions between these two species.

Herren et al. studied the association between Spiroplasma and the fly Drosophila melanogaster. Under normal conditions, Spiroplasma only reduces the life span of the infested fly. This indicates that Spiroplasma has a low impact on the general fitness of its host, only negatively affecting the survival and egg laying ability of old flies. When flies had limited access to nutrients, the number of Spiroplasma they carried was reduced, without the flies losing fitness. This suggests that Spiroplasma growth is dependent on something in the flies' diet.

To understand which nutrients are important for the growth of Spiroplasma in Drosophila, Herren et al. analyzed the hemolymph of flies and found that there are fewer fatty-molecules, called lipids, when nutrients are limited. Healthy flies carrying Spiroplasma also have fewer lipids in their hemolymph, suggesting that these are what Spiroplasma feed on. Indeed, inactivating a protein required by the fly to transport lipids to the hemolymph reduced the growth of Spiroplasma in these flies.

Herren et al. concluded that the growth of Spiroplasma inside its host is limited by the availability of lipids in the hemolymph. Since this is dependent on diet, the dependence on lipids couples the growth of Spiroplasma to the nutritional state of its host. Herren et al. speculate that this mechanism reduces the fitness cost of harboring the microbes and prevents the damaging consequence of an uncontrolled proliferation of the microbes. Moreover, Spiroplasma's preference for lipids may explain why it helps to protect flies against parasitic infection, as many parasites also rely on lipids for their growth. Herren et al. suggest this strategy could also be used in other animal-microbe associations.

DOI: 10.7554/eLife.02964.002

the metabolism of endosymbiotic bacteria is challenging, due to the high level of integration and interdependence between endosymbionts and their hosts. Therefore, despite a general, genomecentric understanding of the metabolic capacities of numerous endosymbionts, little is known about the nature of specific metabolites required for endosymbiont proliferation and the implications of metabolite acquisition by endosymbionts on host physiology and fitness.

Strict maternal transmission is expected to result in the evolution of endosymbionts that have minimized host fitness costs (Werren and O'Neill, 1997). Experimental studies are generally in line with this prediction, for example Wolbachia and Spiroplasma have relatively minor effects on host fitness (Martins et al., 2010; Unckless and Jaenike, 2012), however, fitness costs usually become apparent as hosts age (Ebbert, 1991; Min and Benzer, 1997; Fry et al., 2004). For endosymbionts that colonize the germline from the adult soma, it has been demonstrated that endosymbiont titers are positively correlated with transmission fidelity (Dyer et al., 2005; Unckless et al., 2009). In general, endosymbiont titers (and hence proliferation rates) will therefore be determined by a compromise between the need to minimize host fitness costs and maximize transmission fidelity (Jaenike, 2009). Since host fitness costs are most likely minimized by the limiting excessive endosymbiont proliferation, the factors that limit endosymbiont proliferation are of central importance for the biology of endosymbionts; however, few mechanisms that are capable of limiting endosymbiont proliferation have been identified (Login et al., 2011). The proliferation of bacteria is often controlled by host immune systems; however, it is notable certain endosymbionts, including Spiroplasma, are not susceptible to 
host immune responses (Herren and Lemaitre, 2011) suggesting that other factors are likely to be of importance for limiting their proliferation.

In this study, we used the genetically tractable insect, Drosophila melanogaster and its endosymbiotic Spiroplasma (MSRO strain) to analyze the mechanisms that govern Spiroplasma proliferation and the effects of endosymbiont proliferation on host physiology. We find that under normal rearing conditions MSRO Spiroplasma (henceforth referred to as Spiroplasma) shortens the life span of its host, D. melanogaster. Interestingly, under nutrient limitation, where increased competition between Spiroplasma and its host could be expected, Spiroplasma proliferation is compromised with minimal effect on host fitness. We noted that under nutrient limitation, host hemolymph lipids decline significantly and that under normal rearing conditions, the first observable effect of Spiroplasma-infection on host physiology is a depletion of host lipids. We then used RNAi-based strategies to reduce the hemolymph lipid concentration and find that this inhibits Spiroplasma proliferation and extends the life span of Spiroplasmaharboring flies. We therefore demonstrate that: (i) specific hemolymph lipids are utilized by Spiroplasma and (ii) the availability of hemolymph-lipids limits the proliferation of Spiroplasma.

\section{Results}

\section{The implications of harboring Spiroplasma on host fitness}

We investigated the impact of harboring Spiroplasma on its host's fitness by measuring survival and egg production in both virgin and mated Spiroplasma-infected and uninfected female flies. When maintained on a rich Drosophila diet, flies-harboring Spiroplasma have a significantly shortened life span compared to flies that do not harbor Spiroplasma (Figure 1A, Figure 1-figure supplement 1A), which is in agreement with a previous study on Drosophila willistoni and WSRO Spiroplasma (Ebbert, 1991). Notably, the presence of Spiroplasma did not significantly affect the death rate until flies were about 21-25 days old but flies began to exhibit signs of Spiroplasma-induced pathology between 14 and 21 days, as demonstrated by decreased performance in climbing assays (Figure 1B). Prior to death, aged Spiroplasma-harboring flies exhibit an apparent lack of coordination and tremors. The increase in Spiroplasma-induced lethality and pathology in old flies correlates with the increase of Spiroplasma titers, observed by GPCR of whole flies (Figure 1C) and fluorescence microscopy of hemolymph (Figure 1D). Consistent with previous studies on other Drosophila-endosymbiotic Spiroplasma strains (Anbutsu and Fukatsu, 2003; Haselkorn et al., 2013), we noticed that Spiroplasma titers reached a plateau in old flies (age $>28$ days) suggesting that a factor might limit Spiroplasma proliferation at this stage. In light of the finding that Spiroplama reduces fly life span and the apparent trade-off between life span and reproductive output in Drosophila (Partridge et al., 1987; Sgrò and Partridge, 1999), we also compared the rate of egg production between Spiroplasma-infected and uninfected females. There was a twofold increase in the number of eggs laid by Spiroplasma-infected virgin flies compared to Spiroplasma-uninfected virgin flies over 14 days (Figure 1E). The number of eggs laid by Spiroplasma-infected mated flies over a 14-day period was similar to Spiroplasmauninfected mated flies, however, Spiroplasma-infected mated flies laid an increased number of eggs in the first 2 days post-eclosion (Figure 1-figure supplement 1B). These results indicate that the presence of Spiroplasma stimulates egg production in virgin flies and also in mated flies over the first 2 days post eclosion, while causing a minor decline in egg production of mated flies at later time points. Collectively, these experiments demonstrate that (i) Spiroplasma has a low impact on the general fitness of its host, negatively affecting survival and egg laying only in old flies and (ii) that this decrease in host fitness correlates with higher Spiroplasma titers.

\section{Effects of nutrient limitation on host survival and Spiroplasma proliferation}

We had initially speculated that the competition for resources between Spiroplasma and its host would be more conspicuous upon nutrient scarcity and that under these conditions Spiroplasma might have a more detrimental effect on host fitness. To test this hypothesis, we maintained adult flies on a nutrient poor diet. Under these nutrient-limiting conditions, Drosophila survival is significantly compromised, however it is important to note that this diet still contains sufficient nutritional content to support an entire Drosophila life-cycle (Vijendravarma et al., 2012). It is also worth mentioning that we only examined the effects of nutrient deprivation in adult flies and that for all the experiments conducted Drosophila larvae were raised under normal conditions. Surprisingly, Spiroplasma-infected and uninfected virgin flies had a similar life span (Figure 2A) and produced similar numbers of eggs under 


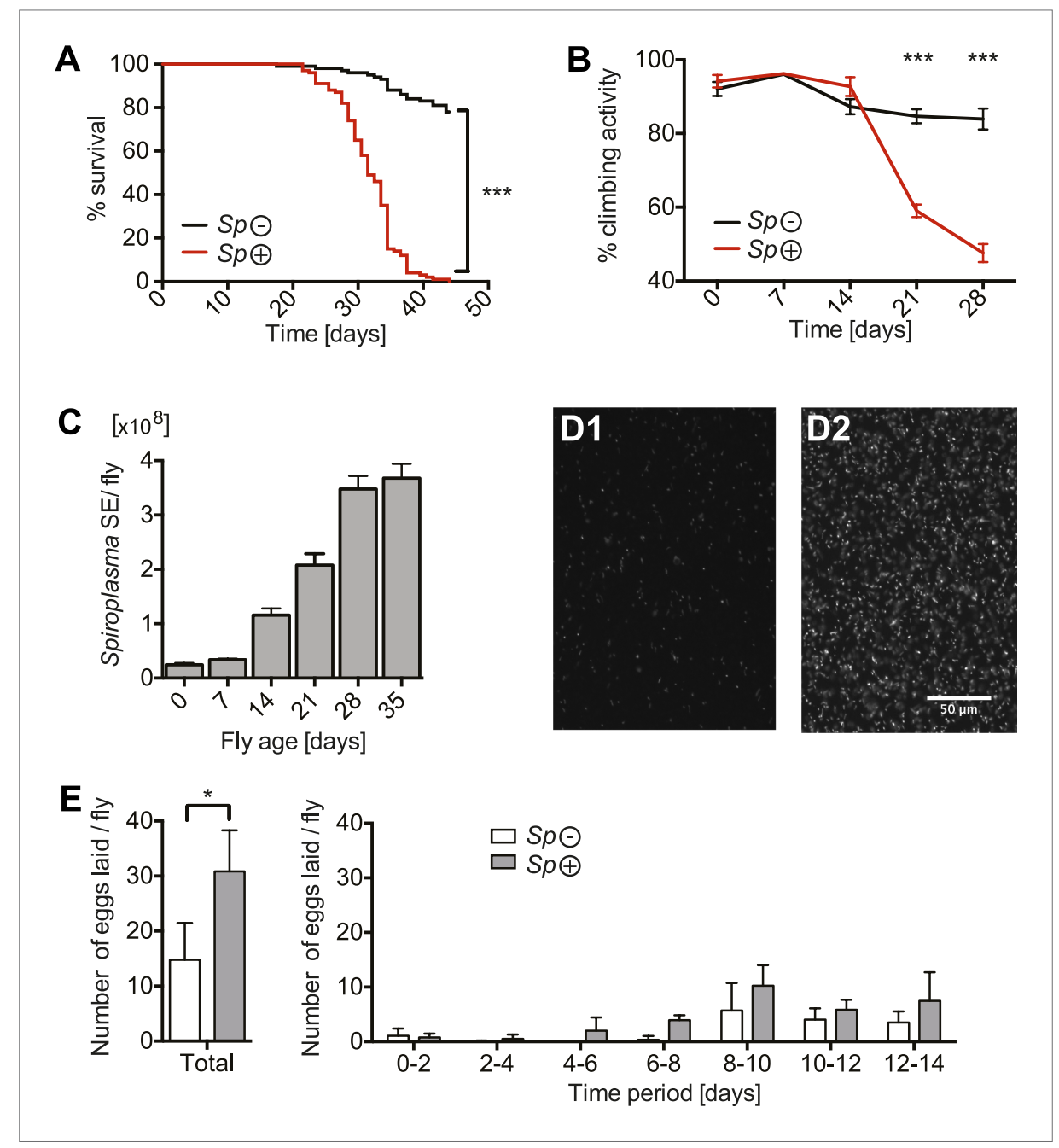

Figure 1. Spiroplasma proliferation is associated with life span reduction. (A) Life span of virgin flies-harboring Spiroplasma (Sp (+)) relative to control flies that do not harbor Spiroplasma (Sp (-)) when kept on a rich Drosophila diet. ${ }^{* *} p<0.0001, N=50$ flies per condition. Shown is one representative experiment out of three independent experiments. (B) The climbing activity of virgin flies-harboring Spiroplasma $(S p(+))$ relative to uninfected flies $(S p(-))$ over time. ${ }^{* * *} p<0.0001, N=20$ flies per condition. Shown is one representative experiment out of three independent experiments. (C) qPCR quantification of the titers of Spiroplasma in virgin flies over aging. Values for each timepoint have at least three samples (five flies pooled per sample). Shown is one representative experiment out of three independent experiments. (D) Fluorescent microscopy images depicting SYTO-9 stained Spiroplasma in Drosophila hemolymph at 7 days (D1) and 21 days (D2) of fly age. (E) The number of eggs laid by virgin flies-harboring Spiroplasma (Sp (+)) relative to control flies that do not harbor Spiroplasma (Sp (-)), in total over 14 days (left panel) and in 2-day period over 14 days (right panel). In total, Spiroplasma-infected virgin flies laid significantly more eggs. ${ }^{*} p=0.02$. Shown is the mean \pm SEM of data pooled from four independent experiments, $\mathrm{N}=20$ flies per experiment.

DOI: 10.7554/eLife.02964.003

The following figure supplement is available for figure 1:

Figure supplement 1. The impact of Spiroplasma infection on survival and egg production by mated females on rich media.

DOI: 10.7554/eLife.02964.004

nutrient-limiting conditions (Figure 2B). For mated flies under nutrient limitation, harboring Spiroplasma resulted in a minor reduction in survival relative to flies that did not harbor Spiroplasma (Figure 2figure supplement 1A). Spiroplasma-infected mated flies produced more eggs than Spiroplasmauninfected mated flies under nutrient-limiting conditions (Figure 2-figure supplement 1B). These findings suggest that, in contrast to our initial hypothesis, harboring Spiroplasma has a rather limited 


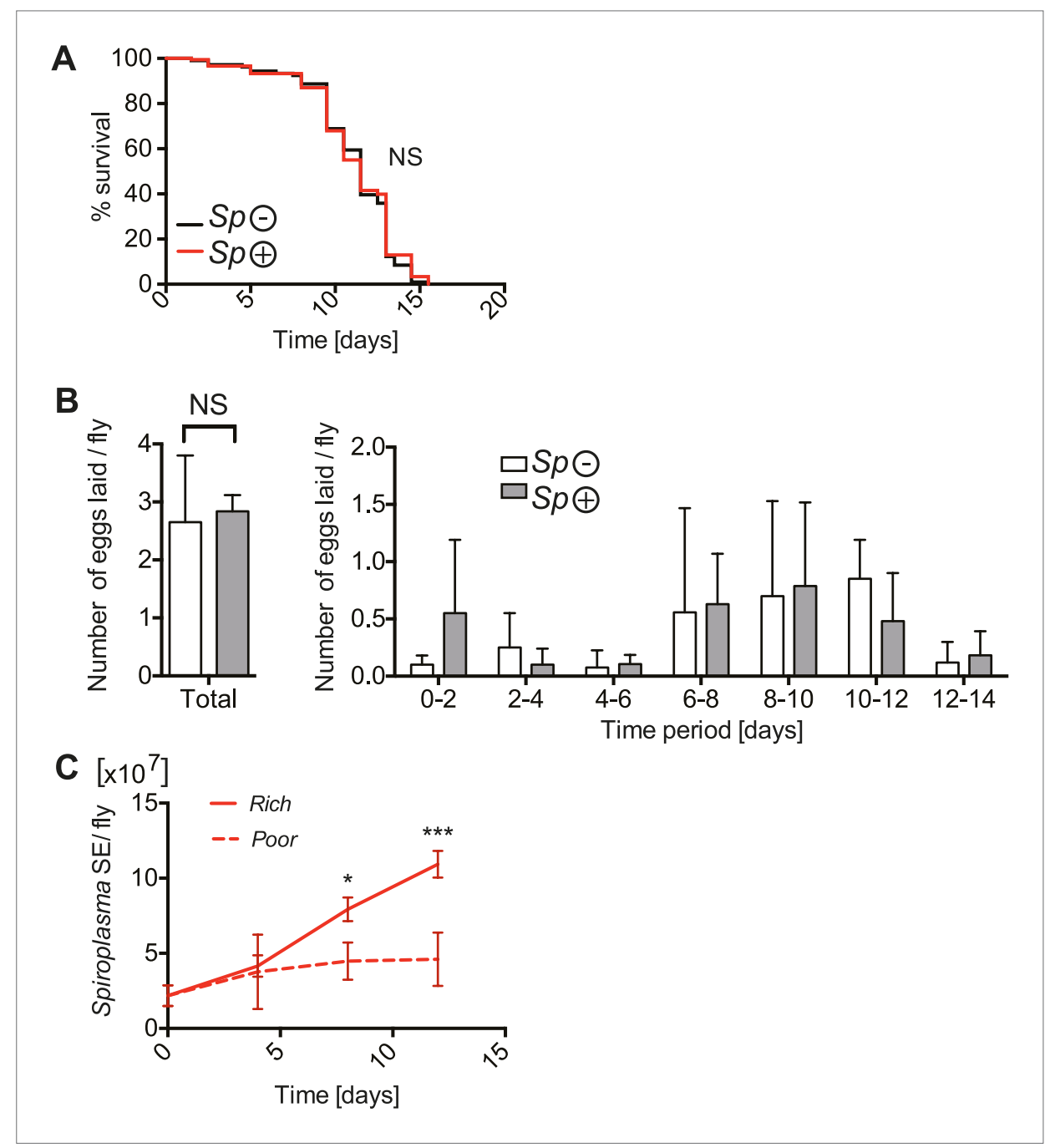

Figure 2. The implications of harboring Spiroplasma under host nutritional depravation. (A) Survival of virgin flies on a nutritionally poor diet. Flies-harboring Spiroplasma $(S p(+))$ do not have significantly different mortality from flies that do not harbor Spiroplasma (Sp (-)). NS ( $p=0.9378)$. N = 50 flies per condition. Shown is one representative experiment out of three independent experiments. (B) The number of eggs laid by virgin flies-harboring Spiroplasma $(S p(+))$ relative to control flies that do not harbor Spiroplasma (Sp (-)), in total over 14 days (left panel) and in 2-day period over 14 days (right panel). Overall, there is no significant difference in the number of eggs laid between Spiroplasma-infected and uninfected virgin flies under nutrient deprivation. NS ( $p=0.77$ ). Shown is the mean \pm SEM of data pooled from four independent experiments, $N=20$ flies per experiment. (C) Quantification of Spiroplasma titers by qPCR reveals that virgin female flies maintained on the same nutritionally poor diet as in panel $\mathbf{A}$ have significantly lower Spiroplasma titers after 8 and 12 days than flies maintained on a rich diet. ${ }^{*} p=0.015$ and ${ }^{* * *} p=0.0002$, respectively. Values for each time-point have at least three samples (five flies pooled per sample). Shown is the mean \pm SEM of one representative experiment out of the three independent experiments that were conducted.

DOI: 10.7554/eLife.02964.005

The following figure supplements are available for figure 2:

Figure supplement 1. The effects of nutrient deprivation on survival and egg production of Spiroplasma-infected mated females.

DOI: 10.7554/eLife.02964.006

Figure supplement 2. Spiroplasma titers in fly hemolymph under nutrient deprivation.

DOI: 10.7554/eLife.02964.007 
fitness cost under nutrient-limiting conditions. Importantly, we observed that Spiroplasma proliferation in whole flies (Figure 2B) and hemolymph (Figure 2-figure supplement 2) was also significantly inhibited when flies are maintained on a nutrient poor diet. Thus, the inhibition of Spiroplasma proliferation in flies that are maintained under nutrient-limiting conditions could explain why Spiroplasma has limited fitness costs under these conditions. For consistency, and to facilitate the maintenance of flies under identical conditions, subsequent experiments were carried out on virgin females (unless otherwise specified).

\section{Spiroplasma proliferation is influenced by the nutrient composition of the diet}

The results above suggest that Spiroplasma proliferation might be dependent on the availability of host factors that are nutritional state-dependent. To identify these factors, we examined the effect that maintaining uninfected flies for 12 days on a nutrient poor diet had on the concentration of metabolites in the hemolymph (where Spiroplasma reside). We found that raising flies on a nutrient poor diet resulted in significantly lower concentrations of protein, sterol and diacylglyceride (DAG, the main transport lipid in Drosophila) (Figure $3 A-C$ ), while levels of glucose and trehalose were not changed significantly (Figure 3-figure supplement $1 A, B$ ) and L-amino acids increased (Figure 3-figure supplement 1C). Thus, nutrient limitation led to a specific decline in hemolymphatic protein and lipid concentrations. We then complemented the nutrient poor diet with either inactivated yeast (rich in protein and lipids) or sucrose, we found that only inactivated yeast extract was able to recover the Spiroplasma proliferation rates observed on rich media (Figure 4). This indicates that Spiroplasma proliferation is not only affected by the caloric content of the food but by the composition of the diet. Taken together, these results lend support to the hypothesis that Spiroplasma proliferation is heavily dependent on hemolymph metabolite composition and that certain metabolites (e.g., lipids and protein) could play a more important role than others (e.g., sugars).

\section{Spiroplasma proliferation depletes lipids from the hemolymph and fat body}

To gain a better insight into the relationship between Spiroplasma and host physiology, we monitored the effects of harboring Spiroplasma on the concentration of metabolites in the hemolymph of flies raised on a rich diet. We observed that the concentration of measured sugars was not changed and that the L-amino acid concentration was even increased in the presence of Spiroplasma (Figure 5A-C). We also found that the hemolymph of flies-harboring Spiroplasma had an increased concentration
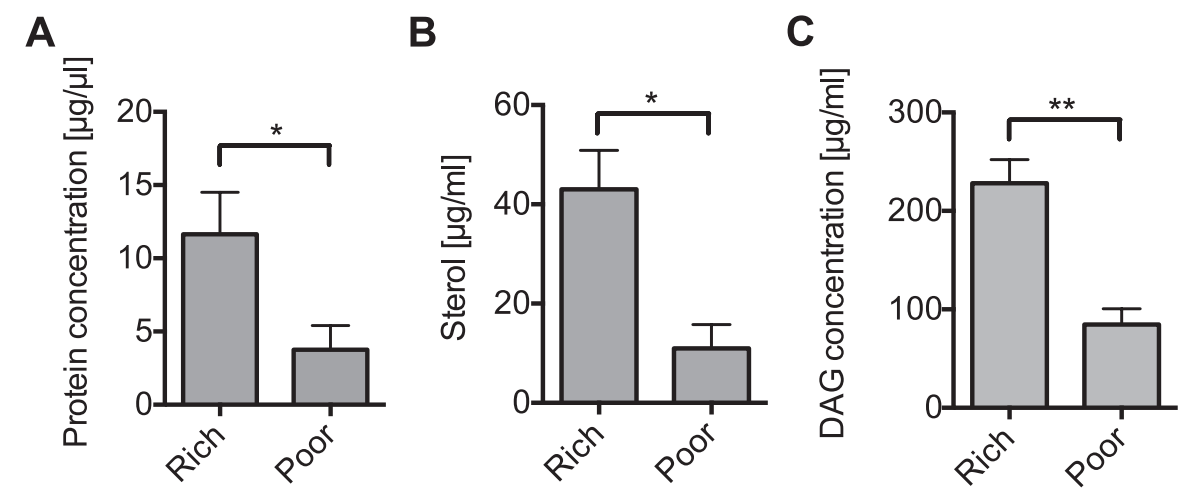

Figure 3. Nutrient deprivation depletes host lipids. (A-C) The protein (A), sterol (B), and DAG (C) concentration of the hemolymph of flies maintained on nutritionally poor diets for 12 days is significantly lowered relative to flies maintained on a nutritionally rich diet. Mean \pm SEM of three independent experiments is shown, ${ }^{\star} p=0.038,{ }^{*} p=0.023$, and ${ }^{* *} p=0.0018$, respectively. DOI: 10.7554/eLife.02964.008

The following figure supplement is available for figure 3 :

Figure supplement 1. The impact of nutrient deprivation on hemolymph metabolite concentrations. DOI: 10.7554/eLife.02964.009 


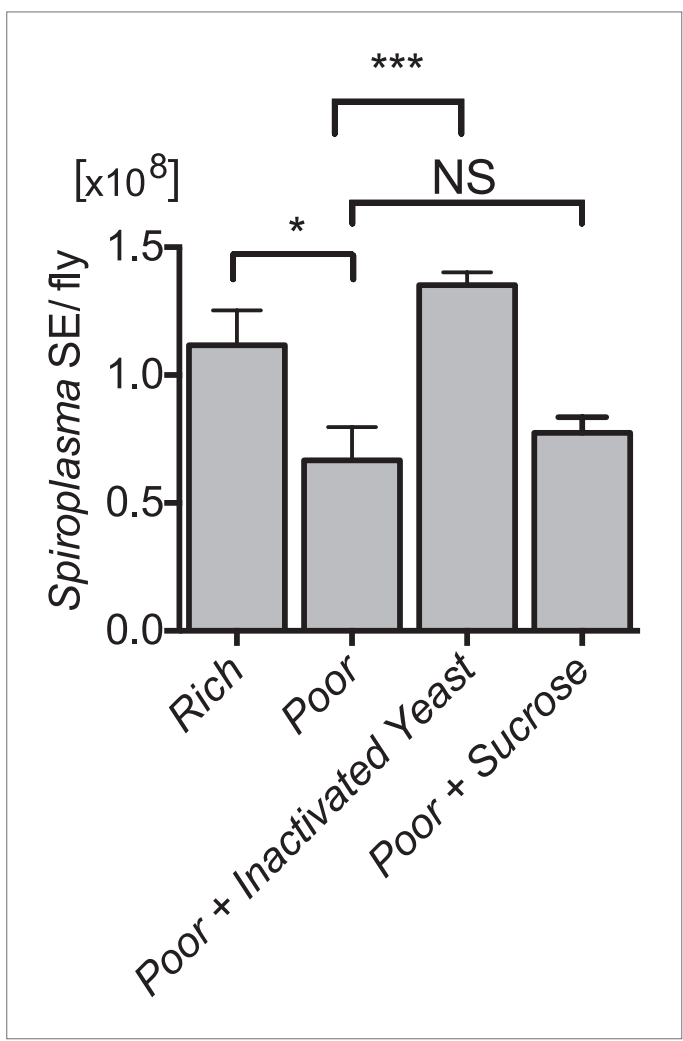

Figure 4. Spiroplasma proliferation is influenced by the nutrient composition of the diet. Quantification of Spiroplasma titers by qPCR reveals that complementing nutrient poor media with inactivated yeast results in a significant increase in Spiroplasma titers after 12 days. ${ }^{* * *} \mathrm{p}=0.0003$. In contrast, complementing nutrient poor media with sucrose does not significantly increase Spiroplasma titers. NS ( $p=0.5)$. Values are the mean \pm SEM of at least four samples (five flies per sample). Shown is one representative experiment out of the three independent experiments that were conducted. DOI: 10.7554/eLife.02964.010

(Figure 5-figure supplement 2). Consistent with the decrease in TAG reserves, 12-day-old Spiroplasma-infected flies succumb more rapidly to acute starvation, in which flies are only given a source of water but no source of nutrition (Figure $5 H$ ). Since TAG stored in the fat body largely derived from hemolymph DAG (Canavoso et al., 2001), the decrease of TAG is likely to be the outcome of the depletion of hemolymphatic DAG by Spiroplasma. Thus, our analyses show that the proliferation of Spiroplasma in flies is associated with a specific depletion of hemolymph DAG as well as a decrease in the amount of fat body lipid storage. These findings, together with the observation that DAG is depleted under nutrient deprivation, suggest that DAG availability limits Spiroplasma proliferation.

\section{Spiroplasma proliferation is associated with the production of cardiolipin}

Since the hemolymph derived from Spiroplasma-infected flies contained Spiroplasma and had reduced DAG, it is likely that Spiroplasma is not directly incorporating DAG into their membrane but rather metabolize DAG into another compound. The main classes of lipids that are present in Spiroplasma are phosphatidylglycerols, sterols, sphingolipids, and cardiolipins (Freeman et al., 1976). Cardiolipins are a class of lipids found exclusively in eubacteria and in the mitochondria of eukaryotic cells (Hawthorne and Ansell, 1982). In contrast to other major lipidic components of Spiroplasma membranes, cardiolipins are not present at detectable levels in the Drosophila hemolymph (Carvalho et al., 2012). 


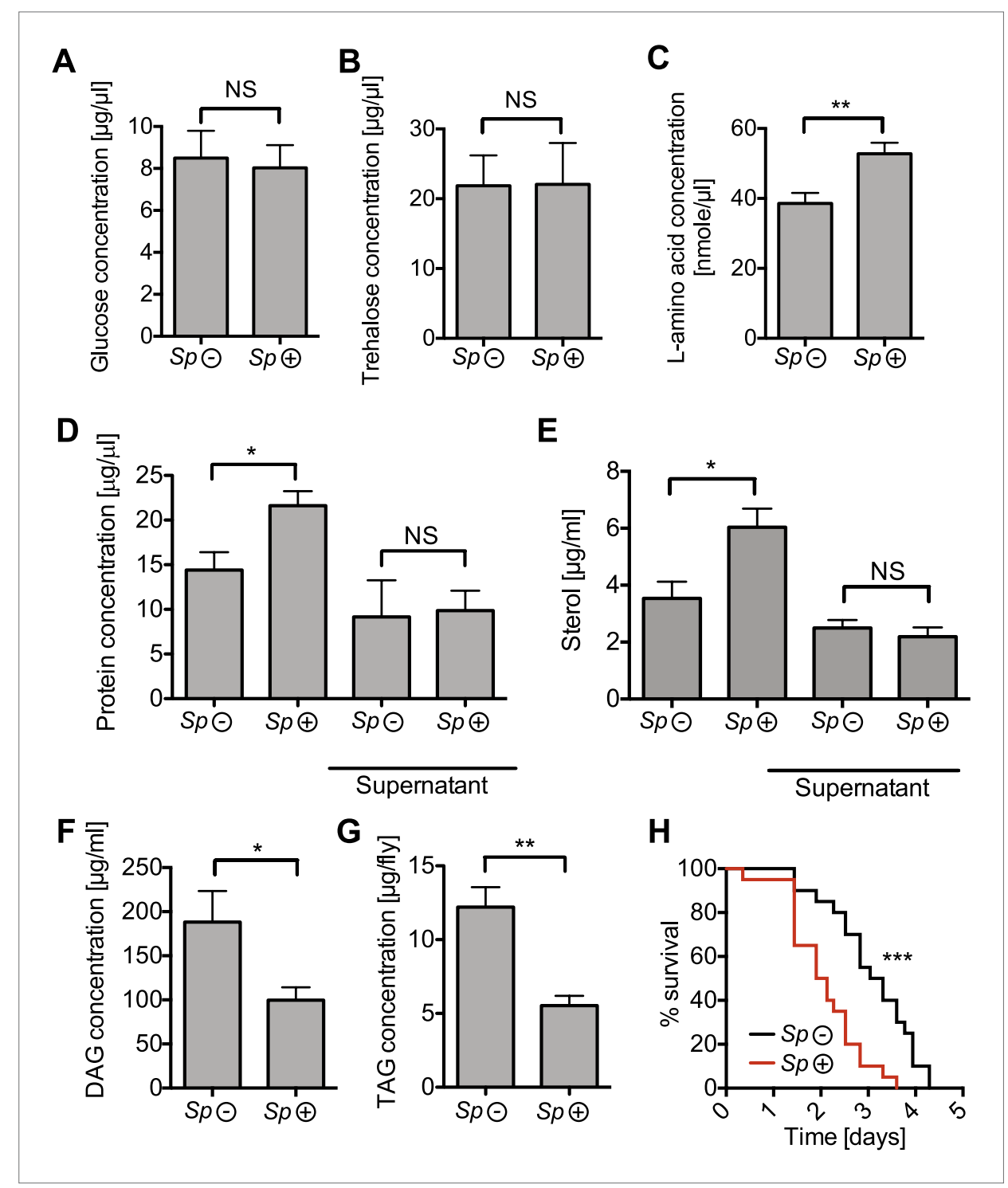

Figure 5. Spiroplasma infection depletes lipids of Drosophila maintained under normal conditions. Quantification of metabolites in flies that have been maintained on rich media for 12 days. Glucose (A), trehalose (B), and L-amino acid (C) concentration within the hemolymph of uninfected flies (Sp (-)) and Spiroplasma-infected flies (Sp (+)). $\mathrm{L}$-amino acid concentration in the hemolymph is significantly higher in Spiroplasma-infected flies while glucose and trehalose concentrations remain unchanged. Mean \pm SEM of three independent experiments is shown, NS $(p=0.798$ and $p=0.977)$ and ${ }^{* *} p=0.0056$. (D-E) Quantifications of protein (D) and sterol (E) concentration in hemolymph from flies that harbor Spiroplasma $(S p(+))$ and uninfected flies (Sp (-)). Hemolymph samples denoted as 'supernatant' have been subjected to an additional centrifugation to remove Spiroplasma cells, whereas all other hemolymph samples contain both Spiroplasma cells and hemolymph. Flies-harboring Spiroplasma have significantly higher total levels of protein and sterol in the hemolymph. Mean \pm SEM of three independent experiments is shown, ${ }^{\star} p=0.04$ and ${ }^{*} p=0.037$. After centrifugation to remove bacteria from the hemolymph, there was no longer any significant difference in protein and sterol concentrations between Spiroplasma-infected and uninfected hemolymph. Mean \pm SEM of three independent experiments is shown, $N S(p=0.881$ and $p=0.491$, respectively). (F) Quantification of DAG content of hemolymph extracts from flies that harbor Spiroplasma (Sp (+)) and flies that do not $(S p(-))$. ${ }^{\star} p=0.0266$. Mean \pm SEM of three independent experiments is shown. (G) Quantification of whole-fly (reflecting mainly fat body) TAG levels in flies that harbor Spiroplasma (Sp (+)) and uninfected flies (Sp $(-)) .{ }^{* *} p=0.0043$. Mean \pm SEM of three independent experiments is shown. (H) Survival of flies subjected to an acute starvation after being maintained on rich media for 12 days. Flies-harboring Spiroplasma (Sp (+)) have Figure 5. Continued on next page 
Figure 5. Continued

significantly greater mortality rate than flies that do not harbor Spiroplasma $(S p(-)) .{ }^{\star \star \star} p<0.0001 . \mathrm{N}=20$ flies per condition, shown is one representative experiment out of three independent.

DOI: 10.7554/eLife.02964.011

The following figure supplements are available for figure 5:

Figure supplement 1. The impact of Spiroplasma proliferation hemolymph DAG concentration in mated flies and old flies.

DOI: 10.7554/eLife.02964.012

Figure supplement 2. The impact of Spiroplasma proliferation on fat body glycogen stores.

DOI: 10.7554/eLife.02964.013

Cardiolipins are comprised of four acyl chains, two phosphate groups and three glycerols, and therefore are made up of similar components to DAGs, which consist of two acyl chains and a glycerol. Analysis of the Spiroplasma genome reveals that Spiroplasma is nearly devoid of lipid metabolic capacities but possess genes involved in the synthesis of cardiolipin from the precursor DAG through a pathway involving DAG-3-phosphate and cytidine diphosphate-DAG (unpublished data). To investigate the production of cardiolipin by Spiroplasma, we conducted a MALDI time-of-flight mass spectrometry (MALDI-Tof-MS) analysis of lipid species in fly hemolymph and found that there were only peaks at $\mathrm{m} / \mathrm{z}$ values that correspond to cardiolipin in the hemolymph of flies-harboring Spiroplasma (Figure 6A). A major ion at $\mathrm{m} / \mathrm{z} 1425.05$ was isolated and fragmented, which confirmed the presence of cardiolipin and revealed that the cardiolipins were comprised of $C 16: 0$ and $C 18: 1$ acyl chains (Figure 6B). In addition, we used liquid chromatography-tandem mass spectrometry (LC-MS/MS) to quantify the effect of Spiroplasma infection on the concentration of individual DAG species in Drosophila hemolymph. We observed that overall DAG concentration declined by $16.3 \%$, however certain DAG species (e.g., C32:1 and C34:1 DAG) declined to a much greater extent (Table 1). It is notable that DAG species that decline to the greatest extent in the presence of Spiroplasma are those likely to contain (based on Drosophila fatty acid composition) one saturated (e.g., C14:0 or C16:0) and one mono-unsaturated (e.g., C16:1 or C18:1) medium-length acyl chain (Shen et al., 2010). The observation that Spiroplasma-generated cardiolipin contains the same configuration of one saturated and one mono-unsaturated medium-length acyl chain (predominately C16:0, C18:1) indicates that Spiroplasma most likely produce cardiolipin using fly hemolymph DAG. The transformation of DAG into cardiolipin offers an explanation for the observed decrease in hemolymph DAG levels in the presence of Spiroplasma.

\section{Spiroplasma acquires hemolymph-lipids prior to storage in the fat body}

In Drosophila, dietary lipids are broken down in the gut lumen by lipases prior to absorption by intestinal cells (Sieber and Thummel, 2012). In the enterocytes, these compounds are used for the synthesis of DAG, which is packaged together with phosphoethanolamine, sterol, other minor lipids, and the apolipophorin protein (Lpp), to form lipoprotein particles. Lpp is produced in the fat body but travels to the gut where it gets loaded with lipids prior to trafficking throughout the body (Palm et al., 2012). Lpp is the main hemolymph lipid carrier, since more than $95 \%$ of the hemolymph lipids in Drosophila co-fractionate with Lpp (Palm et al., 2012).

Our results are consistent with Spiroplasma subverting and utilizing the lipids contained in hemolymph lipoprotein particles prior to their arrival at the fat body. This has the consequence of decreasing the observed levels of stored lipids. To rule out the possibility that Spiroplasma induces the mobilization of lipid stores, which could also decrease TAG levels, we quantified the effect of Spiroplasma on TAG levels in AKHR and Bmm double mutant flies. Adipokinetic hormone receptor (AKHR) and the Brummer lipase $(\mathrm{Bmm})$ are components of two independent pathways that mobilize lipids from Drosophila fat bodies. Signaling through AKHR initiates mobilization of stored lipid in the insect fat body (Gäde and Auerswald, 2003), and Bmm is a TAG lipase involved in mobilization of stored lipid (Grönke et al., 2005). AKHR'; bmm double mutant flies exhibit an 'obese' phenotype because the fat bodies of these flies store lipids but are not subsequently able to mobilize or release lipids (Grönke et al., 2007). We found that fat body TAG levels in $A K H R^{1} ; b m^{1}$ double mutants that harbored Spiroplasma were still significantly lower after 12 days than flies with the same genotype that did not harbor Spiroplasma (Figure 7). While we cannot rule out other, as of yet uncharacterized, lipid mobilization pathways that could theoretically be activated by Spiroplasma, these results are still a strong 


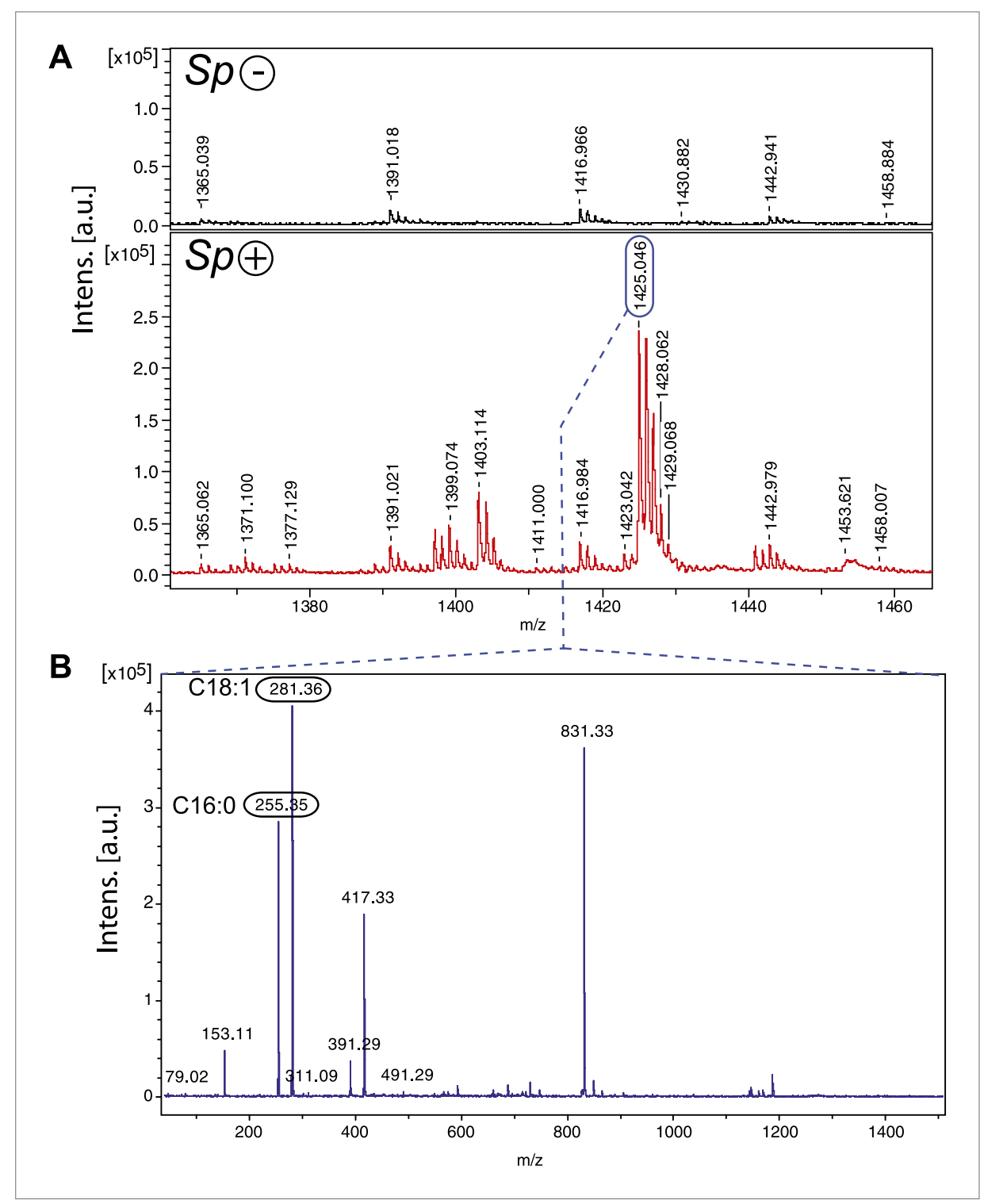

Figure 6. Spiroplasma produces cardiolipin in Drosophila hemolymph. (A) Negative MALDI-TOF/MS lipid profile of hemolymph from Spiroplasma-uninfected (top) and Spiroplasma-infected flies (bottom). The $\mathrm{m} / \mathrm{z}$ signal peaks in the 1380-1460 range of Spiroplasma-uninfected hemolymph do not correspond to $\mathrm{m} / \mathrm{z}$ values of cardiolipin, whereas the peaks in this region for Spiroplasma-infected hemolymph profile (e.g., 1403.11, 1425.05) do correspond to cardiolipin. (B) The isolation and fragmentation of the $\mathrm{m} / \mathrm{z} 1425.05$ parent ion resulted in the generation of daughter ions with peaks at $\mathrm{m} / \mathrm{z} 281.36$ and 255.35 that have been characterized as oleic acid (C18:1) and palmitic acid (C16:0) using Lipid MS Predict software with an error tolerance set to 0.1 u.m.a. The peak at $\mathrm{m} / \mathrm{z} 79.02$ detected in the same experiment corresponds to a phosphate ion. Six additional peaks ( $\mathrm{m} / \mathrm{z}$ 153.11, 311.09, 391.29, 417.33, 491.29 and 831.33) were also detected corresponding to phosphatidyl moieties and the cardiolipin 'backbone' (Hsu and Turk, 2006). Altogether, this indicates that the molecular ion corresponds to the cardiolipid species $[\mathrm{M}-2 \mathrm{H}+\mathrm{Na}]^{-} \mathrm{m} / \mathrm{z} 1425.05$.

DOI: 10.7554/eLife.02964.014

indication that Spiroplasma lipid acquisition most likely occurs in the hemolymph before the point of lipid entry into the fat body stores.

\section{Spiroplasma proliferation depends on hemolymph lipid availability}

We have observed that: (i) Spiroplasma's proliferation is affected by host nutrient limitation and (ii) Spiroplasma consumes the hemolymph DAGs. These findings lead us to hypothesize that the proliferation of Spiroplasma could be constrained by the availability of lipids in the hemolymph, which 
Table 1. Characterization and quantification of hemolymph of DAG species

\begin{tabular}{lccc} 
Component name & Sp $-(\mu \mathrm{g} / \mathrm{ml})$ & $S p+(\mu \mathrm{g} / \mathrm{ml})$ & $\%$ Of total mass decline \\
\hline C28:0 DAG & 13.4 & 12.3 & 1.2 \\
\hline C28:1 DAG & 0.8 & 0.6 & 1.2 \\
\hline C30:2 DAG & 0.7 & 0.5 & -1.0 \\
\hline C30:0 DAG & 0.1 & 0.2 & 0.7 \\
\hline C32:3 DAG & 1.2 & 1.1 & 8.2 \\
\hline C32:2 DAG & 23.1 & 21.8 & 30.1 \\
\hline C32:1 DAG & 19.8 & 15.1 & 4.9 \\
\hline C34:2 DAG & 11.5 & 10.8 & 19.3 \\
\hline C34:1 DAG & 9.7 & 6.9 & 5.6 \\
\hline C34:0 DAG & 1.4 & 0.5 & 1.8 \\
\hline C36:4 DAG & 0.8 & 0.5 & 5.3 \\
\hline C36:3 DAG & 2.9 & 2.2 & 9.3 \\
\hline C36:2 DAG & 4.2 & 2.9 & -0.3 \\
\hline C36:1 DAG & 0.8 & 0.9 & 6.9 \\
\hline C36:0 DAG & 1.7 & 0.7 & \\
\hline
\end{tabular}

Quantification of the absolute concentration of individual DAG species in the hemolymph of Spiroplasma-uninfected $(\mathrm{Sp}(-))$ and Spiroplasma-infected (Sp (+)) mated flies by LC-MS/MS. The \% of total mass decline reflects the percentage of the total decline between Spiroplasma-uninfected and infected samples (a total of $15.1 \mu \mathrm{g} / \mathrm{ml}$ or 16.3\%) that can be attributed to each DAG species. It is notable that C32:1 and C34:1 DAG species decline to a greater extent than other common DAG species such as C28:0 and C32:2. This suggests that Spiroplasma is preferentially incorporating DAGs that have one saturated and one mono-unsaturated acyl chain. Notably, C34:1 DAGs are likely to be made up of oleic (C18:1) and palmitic (C16:0) acids, which have exactly the same acyl chains that were identified in Spiroplasma-generated cardiolipins (Figure 6B). DOI: 10.7554/eLife.02964.015

is known to be heavily dependent on the insect nutritional status (Canavoso et al., 2001). To further investigate the lipid-centric metabolic interplay between Spiroplasma and Drosophila, we monitored Spiroplasma proliferation in flies with a reduced capacity for inter-organ lipid transport and hence decreased hemolymph lipid concentrations. A recent study has shown that RNAi-mediated knockdown of Lpp in the fat body resulted in a decrease in circulating lipoprotein particles and a blockage of lipid export from the gut of Drosophila larvae (Palm et al., 2012). Based on these findings, we used a similar RNAi strategy to knockdown Lpp in the fat body of adult flies. In this experiment, we specifically expressed Lpp-RNAi in the fat bodies of adult flies using a flippase-mediated activation strategy (Marois and Eaton, 2007). We established that RNAi-mediated knockdown of Lpp does not significantly decrease the overall levels of protein in the adult hemolymph (Figure 8-figure supplement 1) but does decrease adult hemolymph-lipids, as shown by quantification of hemolymph DAG and sterol (Figure $\mathbf{8 A}, \mathbf{B}$ ). We then investigated the effect of this lipid reduction on the Drosophila-Spiroplasma interaction. We found that under conditions of Lpp knockdown, Spiroplasma proliferation was severely inhibited resulting in lower Spiroplasma titers in flies after 14, 21, and 28 days of aging (Figure 8C). To rule out any possibility that the genetic background of Lpp-RNAi flies was causing the observed decrease in Spiroplasma titers, we quantified Spiroplasma titers in the absence of activation of LppRNAi by heat-shock and found no significant difference between flies containing the Lpp-RNAi construct and those that did not (Figure 8-figure supplement 2A). In addition, we used an independent Lpp-RNAi construct with another fat body specific driver and activation strategy to confirm that the decrease in Spiroplasma titers was specifically caused by RNAi-mediated Lpp knockdown (Figure 8-figure supplement 2B). It is noteworthy that Spiroplasmas are not known to have the capacity to utilize proteins as nutrient sources (Chang and Chen, 1983), supporting the claim that lipids carried by Lpp (and not the Lpp protein itself) are the factors required for the proliferation of Spiroplasma. Another striking effect of the RNAi-mediated knockdown of Lpp was strongly diminished Spiroplasmainduced old age mortality (Figure 8D). 


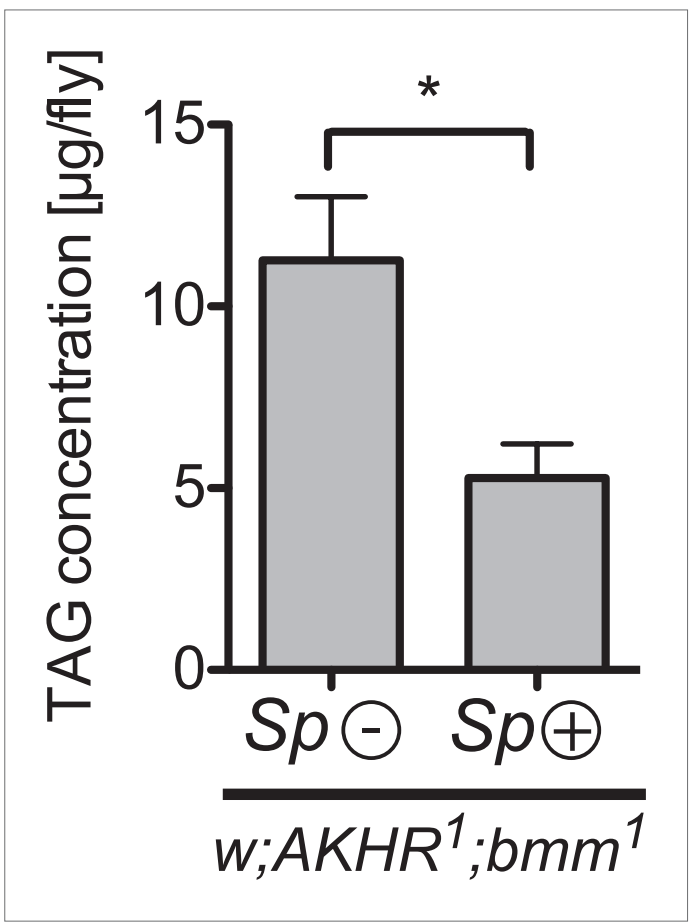

Figure 7. Spiroplasma-induced lipid depletion is not caused by the mobilization of stored lipids. TAG levels in $A K H R^{1} ; B m m^{1}$ double mutants that harbor Spiroplasma $\left(A K H R^{1} ; B m^{1} \mathrm{Sp}(+)\right)$ relative to the same genotype without Spiroplasma (AKHR';Bmm $\mathrm{Sp}(-))$. ${ }^{*} p<0.0153$. Flies were maintained on a rich Drosophila diet for 12 days prior to TAG analysis. Mean \pm SEM of three independent experiments is shown.

DOI: 10.7554/eLife.02964.016

\section{Discussion}

We have demonstrated that Spiroplasma subverts specific host lipids and that its proliferation is limited by the availability of host hemolymph-lipids. This finding is based on several observations: (i) Spiroplasma proliferation rate is decreased in the hemolymph of nutrient deprived flies and old flies, two conditions in which hemolymph-lipid concentration is decreased; (ii) the proliferation of Spiroplasma depletes hemolymph and fat body lipids; and (iii) a genetically induced reduction in Lpp-lipids inhibits Spiroplasma proliferation.

Natural selection is expected to favor vertically transmitted endosymbionts with adaptations that minimize fitness costs to their hosts (Werren and O'Neill, 1997). We hypothesize that Spiroplasma's dependence on hemolymph-lipid availability for proliferation could be of adaptive significance, since it would enable Spiroplasma to limit its proliferation in the face of host nutrient deprivation, and therefore avoid the costly depletion of its host's energy and other vital metabolites. If the proliferation rate of Spiroplasma were to be determined by metabolites that do not decline under nutrient-limiting conditions, for example sugars and L-amino acids, then Spiroplasma proliferation would not be limited under host nutritional deprivation. Such a lack of restriction could result in greater negative effects on its host's fitness. For example, we might expect a striking decrease in reproductive output or survival, which was not observed for Spiroplasma-infected flies maintained

on a nutrient poor diet. We only observed that mated flies-harboring Spiroplasma had increased mortality after about 7 days under nutrient-limiting conditions, and this is not likely to have a major impact on host fitness since increased mortality occurred after the production of eggs had stopped.

Under normal conditions, Spiroplasma's significantly decreases the life span of its host Drosophila (also shown in Ebbert, 1991). However, aspects of this Spiroplasma-induced pathogenesis, including the late onset of symptoms, suggest that Spiroplasma may be employing strategies to minimize host fitness costs. Spiroplasma's capacity to utilize available host lipids for proliferation results in depleted hemolymph-lipids and reduced fat body stores. This depletion also has a fitness consequence for flies, since Spiroplasma-infected flies die more rapidly when subjected to a period of acute starvation, but it is possible that Spiroplasma's reliance on these lipids might be less detrimental than usage of other more critical metabolites that would impact host fitness more directly.

Drosophila fitness and longevity are linked to egg production rates (Partridge et al., 1987), which can be affected by the presence of facultative endosymbionts (Fast et al., 2011). On nutrient rich media, we found that despite increasing early egg production Spiroplasma infection status did not affect the total number of eggs laid over a 14-day period. The Spiroplasma-induced early increase in egg production has been described previously for other Spiroplasma strains (Ebbert, 1991; Martins et al., 2010). While the mechanistic basis of this increase is unknown, it offers a possible explanation for the greater number of eggs laid by Spiroplasma-infected flies on nutrient poor media, where the majority of eggs laid by all flies are in the first 2 days post-eclosion. Under rich nutritive conditions, we observed that Spiroplasma increased by twofold the number of eggs laid by virgin flies over a 14-day period. This finding is suggestive of a Spiroplasma-mediated disruption in the balance between egg retention and laying of virgin flies. One could speculate that Spiroplasma interferes with the signaling pathways (e.g., Juvenile Hormone and/or Ecdysone), which have previously been shown to regulate the production of eggs (Soller et al., 1999). 


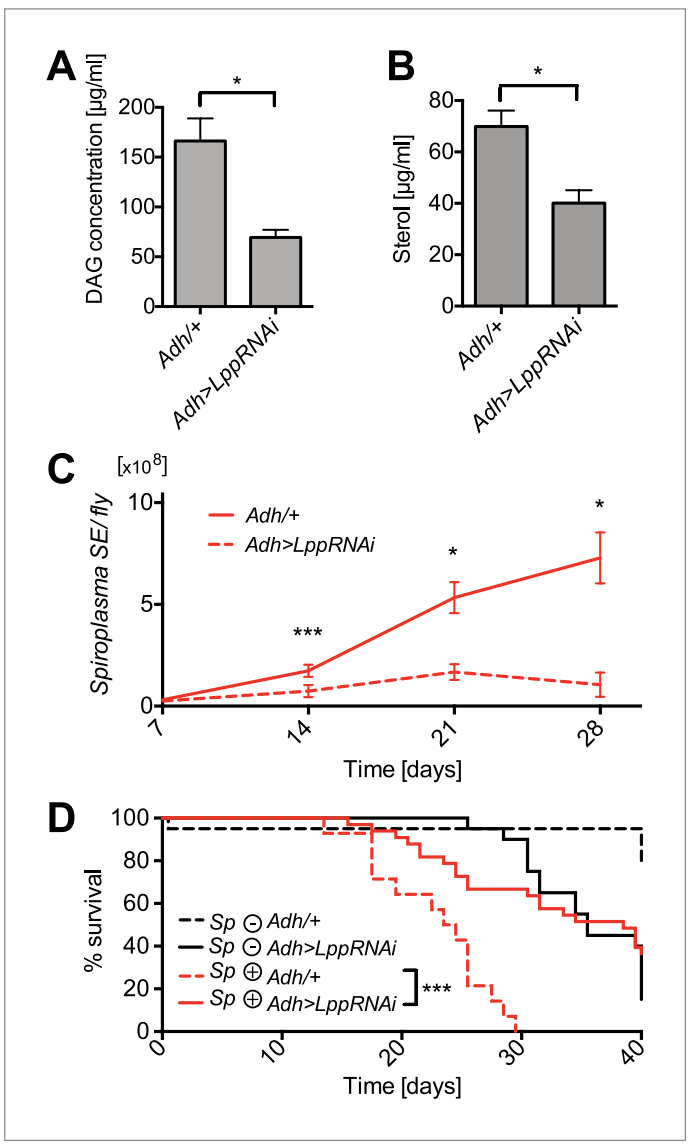

Figure 8. Lpp-lipids are required for Spiroplasma proliferation. (A) Quantification of DAG levels in hemolymph of 12-day-old flies 8 days after knockdown of Lpp by RNAi. Mean \pm SEM of three independent experiments is shown, ${ }^{\star} p=0.015$. (B) Quantification of sterol concentration in hemolymph of 12-day-old flies 8 days after knockdown of Lpp by RNAi. * $p=0.0125$. Mean \pm SEM of three independent experiments is shown. (C) Spiroplasma titers quantified by QPCR in flies which have Lpp expression knocked down by RNAi relative to control flies. Spiroplasma titers were quantified at 7, 14 21 , and 28 days after activation of RNAi. Mean \pm SEM of at least three samples is shown (five flies pooled per sample). ${ }^{* *} p=0.0005$ (14 days), ${ }^{*} p<0.01$ (21 days), and ${ }^{*} p<0.01$ (28 days). Shown is one representative experiment out of three independent experiments. (D) The survival of Spiroplasma-infected (Sp (+)) and Spiroplasmauninfected $(S p(-))$ flies with or without RNAi-mediated Lpp knocked down. ${ }^{* * *} \mathrm{p}<0.0001, \mathrm{~N}=18$ flies per condition. Shown is one representative experiment out of three independent experiments.

DOI: 10.7554/eLife.02964.017

The following figure supplements are available for figure 8 :

Figure supplement 1. Hemolymph protein is not decreased by Lpp depletion. DOI: 10.7554/eLife.02964.018

Figure supplement 2. Spiroplasma titers are decreased by Lpp depletion. DOI: 10.7554/eLife.02964.019
We observed a decrease in Spiroplasma proliferation in flies after 28 days. A late decline in Spiroplasma proliferation has been demonstrated for a number of other Spiroplasma strains (Anbutsu and Fukatsu, 2003; Haselkorn et al., 2013). It is notable that flies aged for 28 days also experience a decline in DAG concentration, which is likely to explain the declining Spiroplasma proliferation rate. We suspect that Spiroplasma proliferation over the life of flies results in elevated Spiroplasma titers that ultimately deplete host hemolymph lipid and constrain further proliferation. In the MSRO-Spiroplasma system, this decline in proliferation does not appear to limit Spiroplasma-induced mortality, however, other Drosophila-Spiroplasma strains appear to reach maximal titers at earlier time points and therefore this is an aspect of the endosymbiont's biology could play a more important role in limiting fitness costs in other systems.

We noted that Spiroplasma-infected flies had higher levels of cardiolipin, suggesting that Spiroplasma synthesize cardiolipin, most likely using DAG acquired from host lipoproteins. We observed that Spiroplasma specifically depleted host DAG species that contained one saturated and one mono-unsaturated acyl chain, which was also the most abundant acyl chain configuration in Spiroplasma-produced cardiolipin (which contained C16:0 and C18:1 acyl chains). The basis of this acyl chain specificity is not known, but it could be linked to biophysical properties of cardiolipins in the highly curved Spiroplasma membrane. The utilization of DAG as a precursor for cardiolipin synthesis is supported by the annotation of the Spiroplasma genome (unpublished data), which enabled the identification of a biosynthetic pathway similar to that described in Mycoplasma synoviae and Mycoplasma hyopneumoniae for the synthesis of cardiolipin from acylglycerols through DAG-3-phosphate and cytidine diphosphate-DAG (Arraes et al., 2007). A number of studies have demonstrated links between cardiolipin and cell death (Gonzalvez and Gottlieb, 2007), which raise the possibility that cardiolipin produced by Spiroplasma could be involved in its pathological effects in old flies.

The other lipids that comprise a smaller fraction of Lpp's cargo, including sterols and sphingolipids (Palm et al., 2012) might also be important for Spiroplasma proliferation but these lipids would most likely be incorporated unchanged into the membrane of Spiroplasma. Of the other lipid classes contained in lipoproteins, sterols are of particular interest as they have been shown to be required for proliferation of all species of 
Spiroplasma, and to be highly abundant in the Spiroplasma plasma membrane (Freeman et al., 1976). Amongst bacteria, this requirement for sterol is unique to mollicutes (Dahl, 1993). Since insects are auxotrophic for sterols (Canavoso et al., 2001), their concentrations are determined by dietary uptake. Although it is therefore possible that the sterol availability could play a key role in coordinating Spiroplasma proliferation with host nutritional state, we were unable to recover normal Spiroplasma proliferation rates when flies were maintained on nutrient poor media complemented with sterol (unpublished data). This finding suggests that, while sterol might be important for Spiroplasma proliferation, it is not sufficient to induce proliferation under conditions of nutrient limitation.

The strategies employed in different insect endosymbioses to limit over-proliferation of endosymbionts are not well characterized. We have proposed a model in which Spiroplasma's dependence on host hemolymph-lipid availability could limit its over-proliferation, primarily in the face of host nutritional scarcity. This mechanism could be important for the controlling proliferation of diverse extracellular endosymbionts. Different mechanisms are likely to be involved in intracellular endosymbiont control. For example, proliferation and localization of the obligate Sitophilus primary endosymbiont (SPE) is controlled by the expression of a specific antimicrobial peptide from its host, Sitophilus weevils (Login et al., 2011). In aphids, studies suggest the activation of the host lysosomal system is involved in controlling titers of the obligate intracellular endosymbiont, Buchnera (Nishikori et al., 2009).

The metabolic exchanges between endosymbiotic bacteria and their arthropod hosts are generally not well understood, partly due to challenges associated with high levels of integration and interdependence between partners. Most of the available information is indirect, coming from the study of endosymbiont genomes, which reveals limited metabolic capacities and high levels of dependence on hosts (Zientz et al., 2004; Gosalbes et al., 2010). There are a number of studies that examined metabolic exchanges between hosts and obligate endosymbionts, which provide their hosts with one or more vital metabolite that is missing from the host's diet (Douglas, 1998; Russell et al., 2013). These studies usually consider metabolic transfers from endosymbiont to host and not in the reverse direction (Dale and Moran, 2006). We have identified a transfer of metabolites from host to endosymbiont, showing that Drosophila Lpp-lipids are used by Spiroplasma for proliferation and more specifically provide evidence indicating that host-derived DAG is converted to cardiolipin by Spiroplasma.

A number of recent studies have revealed that facultative endosymbionts protect their hosts from parasites and pathogens (Oliver et al., 2003; Hedges et al., 2008; Teixeira et al., 2008). Endosymbiotic Spiroplasma has been implicated in a number of cases, including protecting diverse hosts from various eukaryotic parasites including parasitoid wasps, parasitic nematodes, and fungi (Jaenike et al., 2010; Xie et al., 2010; kukasik et al., 2012). We speculate that Spiroplasma-mediated protection could be linked to lipid utilization. Indeed, many parasitoid wasps are unable to synthesize fatty acids and their development requires the acquisition of host lipids (Visser et al., 2010). Thus, sequestration of lipid by Spiroplasma might limit availability to any parasites or pathogens that occupy the same niche.

Here, we have shown that the rate of Spiroplasma proliferation and onset of fly mortality are decreased upon depletion of hemolymph lipids. It is noteworthy that a variety of other microorganisms known to proliferate in Drosophila hemolymph and to cause pathogenesis such as Erwinia carotovora strain 15, Listeria monocytogenes, Candida albicans, and Enterococcus fecalis do not appear to have attenuated virulence when hemolymph lipids are depleted (unpublished data). Many of these pathogens are free-living and are likely to have retained well-developed metabolic capacities (including the capacity to synthesize lipids). It is interesting to speculate that increased reliance on host provision of lipids is part of a suite of adaptations that facilitate the evolution of chronic, low-virulence infection strategies. It is notable that a number of pathogens that have the capacity to form chronic infections in humans including Mycobacterium tuberculosis and Chlamydia trachomatis have been shown to be heavily dependent on lipids acquired from their hosts (Ehrt and Schnappinger, 2007; Robertson et al., 2009). Thus, the findings discussed here for endosymbionts could be of more general importance for host-microbe interactions.

\section{Materials and methods}

\section{Fly stocks and handling}

We used a wild-type Oregon- $R\left(O R^{R}\right)$ fly stock that harbors MSRO Spiroplasma (Pool et al., 2006; Herren and Lemaitre, 2011) but not Wolbachia. The w;AKHR ${ }^{1} ; b^{\prime} m^{1}$ stocks used have been described (Grönke et al., 2007). RNAi-mediated knockdown of Lpp was achieved using a pFRiPE-mediated 
inducible RNAi element (Marois and Eaton, 2007), placed in the presence of a heat-shock inducible flippase and an Adh-GAL-4 driver that is mostly active in the fat body. The strategy and stocks used are analogous to a previously published study, except that we induced RNAi in adults, as opposed to larvae (Palm et al., 2012). We induced the flippase by heat-shock $\left(1.5 \mathrm{hr}\right.$ at $37^{\circ} \mathrm{C}$ in a water bath) in 4- to 5-day-old adult flies. This results in the excision of an upstream spacer region and activation of the UAS-Lpp-RNAi construct driven by Adh-GAL-4 and the silencing of Lpp in the fat body, specifically at the adult stage. An additional strategy used to knockdown the expression of Lpp involved the C564-GAL-4 driver (also mostly active in the fat body) in conjunction with tubulin-gal80ts, a temperature-sensitive repressor of GAL-4 expression, which blocks GAL-4 expression at $18^{\circ} \mathrm{C}$ but not $29^{\circ} \mathrm{C}$ (McGuire et al., 2003). Flies that contained both the C564-GAL-4, tubulin-gal80ts, and UAS-LppRNAi(46) elements were maintained at $18^{\circ} \mathrm{C}$ and then shifted to $29^{\circ} \mathrm{C}$ as adults to induce UAS-LppRNAi(46) and knockdown Lpp expression. The UAS-Lpp-RNAi(46) stock is TRiP \#HM05157 originating from the transgenic RNAi project at the Harvard Medical School. Since Spiroplasma MSRO is vertically transmitted and kills male embryos, Spiroplasma-infected stocks were generated in several steps: (1) Crossing an infected $O R^{R}$ female with males carrying appropriate balancer chromosomes (2) Crossing the balanced female progeny with males of the genotype of interest (either $w ; A K H R ; B m m, w, H S$ FLP;adh-GAL-4/bcg or Dipt-GFP,C564-GAL-4;tub-GAL80ts) (3) Several back-crosses were then carried out, resulting in a homozygous Spiroplasma-infected female with the appropriate genotype. These stocks were then maintained by crossing females with non-infected males of the same genotype. Spiroplasma-infected females carrying a GAL-4 construct were then crossed with males carrying UASRNAi constructs or controls ( $w$ background). All flies were maintained at $25^{\circ} \mathrm{C}$ unless otherwise specified. The density of animals per vial was equilibrated between Spiroplasma-infected and Spiroplasma-uninfected stocks for development under similar levels of larval competition. Unless otherwise specified, all flies used were females and virgins. For survival experiments, counts were made every $24 \mathrm{hr}$ and flies were transferred to new tubes every 3-4 days (2 days for mated flies). Climbing assays were carried out as described (Barone and Bohmann, 2013). Acute starvation assays have been described previously (Grönke et al., 2007). To quantify the number of eggs laid, flies were collected immediately post-eclosion and maintained in individual Drosophila vials that each contained five flies. Eggs were counted every $48 \mathrm{hr}$ over a 14-day period, and the number of eggs laid per $48 \mathrm{hr}$ per fly was calculated (correcting for any fly mortality). For experiments that required mated flies, males (three for every five females) were placed in the Drosophila vials and then removed after 7 days, males that died prior to this were replaced.

\section{Drosophila media}

Flies were raised and maintained on a standard cornmeal-agar diet, referred to as 'rich media'. Normal media contain $4.5 \mathrm{~g}$ agar, $58.8 \mathrm{~g}$ inactivated yeast (Springaline BA95/0; Biospringer, Milwaukee, WI, USA), $35 \mathrm{~g}$ maize flour (Farigel Maize; Westhove, Ennezat, France), $34.8 \mathrm{ml}$ of 1:1 mix of grape and multi-fruit juice (approximately $8.2 \mathrm{~g}$ of sugar), $3.6 \mathrm{ml}$ of propionic acid, and $18 \mathrm{ml}$ of a $10 \%$ solution of methyl paraben in $85 \%$ ethanol per $600 \mathrm{ml}$ of water. For nutrient deprivation, adults were maintained on a restrictive diet referred to as 'poor media', which contains $9 \mathrm{~g}$ agar, $1.9 \mathrm{~g}$ inactivated yeast, $7.5 \mathrm{~g}$ maize flour, $4.5 \mathrm{~g}$ sucrose, $9 \mathrm{~g}$ glucose, $0.3 \mathrm{~g} \mathrm{MgSO} 4,0.3 \mathrm{~g} \mathrm{CaCl}_{2}, 3.6 \mathrm{ml}$ propionic acid, and $18 \mathrm{ml}$ of a $10 \%$ solution of methyl paraben in $85 \%$ ethanol per $600 \mathrm{ml}$ of water (Vijendravarma et al., 2012). Poor media complemented with inactivated yeast contain an additional $34 \mathrm{~g}$ of inactivated yeast. Poor media complemented with sucrose contain an additional $35.5 \mathrm{~g}$ of sucrose. In all cases, larvae were raised on rich media.

\section{Imaging}

To observe Spiroplasma in fly hemolymph, flies were dissected on microscope slides in $5 \mu$ PBS containing 0.02 mM SYTO9 (Invitrogen, Carlsbad, CA, USA). Slides were then mounted and observed on an Axioimager Z1 (Zeiss, Oberkochen, Germany). Images were captured with an Axiocam MRn camera and Axiovision software.

\section{DNA extraction and qPCR}

We extracted DNA from five flies per sample. The DNA extraction and quantitative PCR protocols have been previously described (Anbutsu and Fukatsu, 2003; Herren and Lemaitre, 2011). To determine the absolute number of bacteria per extraction, we extracted infected fly hemolymph and used fluorescence microscopy to calculate the concentration of Spiroplasma cells stained using SYTO9 
(as described above). A dilution series of known concentrations of Spiroplasma cell equivalents (SE) was then combined with five uninfected flies prior to DNA extraction and QPCR, which enabled us to generate a calibration curve. In subsequent analyses, to account for differences between qPCR runs, we always used a positive control of known Spiroplasma concentration. The results for all experiments involving Spiroplasma titers are given in SE per fly, which represents the absolute quantities of Spiroplasma per fly. A host gene, RPS17, was also always quantified to verify the quality of the extraction but did not use this value in the analyses. To quantify bacteria in hemolymph samples, $0.5 \mu$ of hemolymph (collected as described for metabolite analyses) was diluted in $300 \mu \mathrm{l}$ cell lysis buffer prior to DNA extraction and qPCRs as previously described (Herren and Lemaitre, 2011).

\section{Metabolite analyses}

Drosophila hemolymph was collected from flies individually using a Drummond nanoject and pulled capillary needle. Metabolic quantifications are given as the mass $(\mu \mathrm{g})$ of metabolite per $\mu \mathrm{l}$ or $\mathrm{ml}$ of Drosophila hemolymph. For each hemolymph sample, we collected $2 \mu \mathrm{l}$ ( $25 \mathrm{flies})$, which was then diluted in $100 \mu \mathrm{l} \mathrm{H}_{2} \mathrm{O}$ prior to subsequent analyses. For all analyses hemolymph samples were centrifuged at $13,000 \times \mathrm{g}$ for $2 \mathrm{~min}$ to remove Drosophila cells. Protein concentration was determined using a Bradford assay (Bio-Rad, Hercules, CA). Glucose and trehalose concentration was quantified using glucose HK kit (Sigma-Aldrich, St. Louis, MO, USA). Samples were treated with or without trehalase (11 $\mathrm{mU}$, Sigma-Aldrich) overnight at $37^{\circ} \mathrm{C}$, and trehalose levels were obtained by subtracting the amount of free glucose in the untreated sample from the total glucose present in the sample treated with trehalase. L-amino acid concentration was quantified by a coupled enzyme reaction, using the L-amino acid quantitation kit (Sigma-Aldrich). Hemolymph DAG and fat body TAG were analyzed using a coupled colorimetric assay (Hildebrandt et al., 2011). Sterols were quantified using the Amplex Red Cholesterol Assay kit (ThermoFisher Scientific, Waltham, MA, USA), which detects primarily cholesterol but also other sterols found in Drosophila (e.g., ergosterol). For protein and sterol assays, Spiroplasma were removed from hemolymph samples by centrifugation at $15,000 \times g$ for $15 \mathrm{~min}$. For whole fly analyses, 10 flies were homogenized in $250 \mu \mathrm{l}$ PBS and the quantifications are given per fly. Note that there was no significant difference in the mass of virgin flies with and without Spiroplasma at 7 and 14 days of age (unpublished data). There was also no significant difference in the feeding rate of virgin flies with and without Spiroplasma (unpublished data) as measured by CAFE assay (Ja et al., 2007) over a period of $4 \mathrm{hr}$ at 3,10 , and 12 days of age.

\section{MALDI-ToF-MS and MS/MS}

Drosophila hemolymph (collected as described for metabolite analyses) was diluted in 1:10 in $\mathrm{H}_{2} \mathrm{O}$. Lipids were extracted using a mixture of chloroform/methanol (50/50 vol:vol). After mixing and centrifugation, $1 \mu \mathrm{l}$ of the lipid extract was diluted with $1 \mu \mathrm{l}$ of 9-aminoacridine matrix $(25 \mathrm{mg} / \mathrm{ml}$, dissolved in isopropanol/acetonitrile [3/2, vol/vol]). For MALDI-Tof-MS analysis, $1 \mu$ of the mixture was rapidly spotted on an MTP 384 polished steel MALDI plate (Bruker Daltonics, Billerica, MA, USA). MALDI-Tof mass spectra of the Drosophila hemolymph samples were acquired using FlexControl 3.0 (Bruker Daltonics) on a Bruker AutoFlex III Smartbeam (Bruker Daltonics) in a negative reflectron mode at a laser beam attenuation of 50 and focus of 40 at $100 \mathrm{~Hz}$ as laser repetition rate. A total of 1000 shots were acquired in the mass range of 400 to $2000 \mathrm{~m} / \mathrm{z}$. Data were processed with FlexAnalysis 3.0 (Bruker Daltonics). Calibration of the instrument was performed using Peptide Standard Calibration II (Bruker Daltonics). 1000 ion counts were accumulated in the mass spectrometer prior to fragmentation. Fragmentation was performed with the Bruker designed method and at a laser beam attenuation of $42 \%$, a laser repetition rate of $100 \mathrm{~Hz}$ and a reflector detector voltage set to $1.861 \mathrm{kV}$ in a negative mode.

\section{LC-MS/MS}

Hemolymph was extracted from 1250 7-day-old adult Spiroplasma-infected and Spiroplasmauninfected mated female flies by pricking flies in the abdomen and thorax before centrifugation of flies at $16000 \times \mathrm{g}$ at $4^{\circ} \mathrm{C}$ for $30 \mathrm{~min}$ in a $10-\mu \mathrm{m}$ filter spin column (Mobitec, Goettingen, Germany). Hemolymph was subsequently filtered in Ultrafree-MC Centrifugal Filter Units ( $0.22 \mu \mathrm{m}$ pores) at $16000 \times \mathrm{g}$ at $4^{\circ} \mathrm{C}$ for $10 \mathrm{~min}$. These samples were then sent to Avanti Polar Lipids analytical services division (http://www.avantilipids.com) where lipids were extracted (Folch et al., 1957) prior to extracts being spiked with internal standards for LC-MS/MS quantification of diacylglygerol species concentrations (Hutchins et al., 2008). 


\section{Data treatment and statistical analysis}

Statistical significance was calculated using a Gehan-Breslow-Wilcoxon test for survivals and an unpaired Student's $t$ test for all other experiments (with GraphPad Prism 5.0) and considered significant if $p$-values were lower than 0.05 . Asterisks indicate the level of significance: ${ }^{\star} p<0.05,{ }^{\star \star} p<0.01$, and ${ }^{* \star *} p<0.001$ and NS (non-significant).

\section{Acknowledgements}

We would like to thank Laure Beven, Wilhelm Palm, Barbara Herren, Roshan Vijendravarma, Steve Perlman, and Melanie Blokesch for informative discussions and suggestions regarding the manuscript. We would like to thank Suzanne Eaton and Ronald P Kühnlein for donating fly stocks and Ryma Acheraiou for assistance. We thank the TRiP at Harvard Medical School (NIH/NIGMS R01-GM084947) and the Bloomington stock center for providing transgenic RNAi fly stocks used in this study. This work was supported by an ERC Advanced Grant.

\section{Additional information}

Funding

\begin{tabular}{lll} 
Funder & Grant reference number & Author \\
\hline European Research Council & 339970 & Juan C Paredes, \\
& & Fanny Schüpfer, \\
& Bruno Lemaitre \\
\hline
\end{tabular}

The funder had no role in study design, data collection and interpretation, or the decision to submit the work for publication.

Author contributions

$\mathrm{JKH}$, Conceiving the project, Design of experiments, Performing experiments, Analyzing data, Drafting of article; JCP, Design of experiments, Performing experiments, Analysis of data, Drafting of article; FS, $\mathrm{KA}, \mathrm{PB}$, Performing experiments, Analysis of data; BL, Conceiving the project, Design of experiments, Drafting of article

Author ORCIDs

Jeremy K Herren, (iD http://orcid.org/0000-0003-2239-7275

\section{References}

Albertson R, Casper-Lindley C, Cao J, Tram U, Sullivan W. 2009. Symmetric and asymmetric mitotic segregation patterns influence Wolbachia distribution in host somatic tissue. Journal of Cell Science 122:4570-4583. doi: $10.1242 / j c s .054981$.

Anbutsu H, Fukatsu T. 2003. Population dynamics of male-killing and non-male-killing spiroplasmas in Drosophila melanogaster. Applied and Environmental Microbiology 69:1428-1434. doi: 10.1128/AEM.69.3.1428-1434.2003.

Anbutsu H, Fukatsu T. 2006. Tissue-specific infection dynamics of male-killing and nonmale-killing spiroplasmas in Drosophila melanogaster. FEMS Microbiology Ecology 57:40-46. doi: 10.1111/j.1574-6941.2006.00087.x.

Arraes FBM, Carvalho MJA, de Maranhão AQ, Brígido MM, Pedrosa FO, Felipe MSS. 2007. Differential metabolism of Mycoplasma species as revealed by their genomes. Genetics and Molecular Biology 30:182-189. doi: 10.1590/S1415-47572007000200004.

Barone MC, Bohmann D. 2013. Assessing neurodegenerative phenotypes in Drosophila dopaminergic neurons by climbing assays and whole brain immunostaining. Journal of Visualized Experiments 74:e50339. doi: $10.3791 / 50339$.

Canavoso LE, Jouni ZE, Karnas KJ, Pennington JE, Wells MA. 2001. Fat metabolism in insects. Annual Review of Nutrition 21:23-46. doi: 10.1146/annurev.nutr.21.1.23.

Carvalho M, Sampaio JL, Palm W, Brankatschk M, Eaton S, Shevchenko A. 2012. Effects of diet and development on the Drosophila lipidome. Molecular Systems Biology 8:1-17. doi: 10.1038/msb.2012.29.

Chang CJ, Chen TA. 1983. Nutritional requirements of two flower spiroplasmas and honeybee spiroplasma. Journal of Bacteriology 153:452-457.

Dahl J. 1993. The role of cholesterol in mycoplasma membranes. Subcellular Biochemistry 20:167-188. doi: 10.1007/978-1-4615-2924-8_5.

Dale C, Moran NA. 2006. Molecular interactions between bacterial symbionts and their hosts. Cell 126:453-465. doi: 10.1016/j.cell.2006.07.014. 
David J, Cohet Y, Fouillet P. 1975. The variability between individuals as a measure of senescence: a study of the number of eggs laid and the percentage of hatched eggs in the case of Drosophila melanogaster. Experimental Gerontology 10:17-25. doi: 10.1016/0531-5565(75)90011-X.

Dobson SL, Bourtzis K, Braig HR, Jones BF, Zhou W, Rousset F, O'Neill SL. 1999. Wolbachia infections are distributed throughout insect somatic and germ line tissues. Insect Biochemistry and Molecular Biology 29:153-160. doi: 10.1016/S0965-1748(98)00119-2.

Douglas AE. 1998. Nutritional interactions in insect-microbial symbioses: aphids and their symbiotic bacteria Buchnera. Annual Review of Entomology 43:17-37. doi: 10.1146/annurev.ento.43.1.17.

Duron O, Bouchon D, Boutin S, Bellamy L, Zhou LQ, Engelstadter J, Hurst GD. 2008. The diversity of reproductive parasites among arthropods: Wolbachia do not walk alone. BMC Biology 6:27. doi: 10.1186/1741-7007-6-27.

Dyer KA, Minhas MS, Jaenike J. 2005. Expression and modulation of embryonic male-killing in Drosophila innubila: opportunities for multilevel selection. Evolution 59:838-848. doi: 10.1554/04-527.

Ebbert MA. 1991. The interaction phenotype in the Drosophila willistoni-Spiroplasma symbiosis. Evolution 45:971-988. doi: 10.2307/2409703.

Ehrt S, Schnappinger D. 2007. Mycobacterium tuberculosis virulence: lipids inside and out. Nature Medicine 13:284-285. doi: 10.1038/nm0307-284.

Fast EM, Toomey ME, Panaram K, Desjardins D, Kolaczyk ED, Frydman HM. 2011. Wolbachia enhance Drosophila stem cell proliferation and target the germline stem cell niche. Science 334:990-992. doi: 10.1126/science.1209609.

Folch J, Lees M, Sloane-Stanley GH. 1957. A simple method for the isolation and purification of total lipids from animal tissues. Journal of Biological Chemistry 226:497-509.

Freeman BA, Sissenstein R, McManus TT, Woodward JE, Lee IM, Mudd JB. 1976. Lipid composition and lipid metabolism of Spiroplasma citri. Journal of Bacteriology 125:946-954.

Fry AJ, Palmer MR, Rand DM. 2004. Variable fitness effects of Wolbachia infection in Drosophila melanogaster. Heredity 93:379-389. doi: 10.1038/sj.hdy.6800514.

Frydman HM, Li JM, Robson DN, Wieschaus E. 2006. Somatic stem cell niche tropism in Wolbachia. Nature 441:509-512. doi: 10.1038/nature04756.

Gäde G, Auerswald L. 2003. Mode of action of neuropeptides from the adipokinetic hormone family. General and Comparative Endocrinology 132:10-20. doi: 10.1016/S0016-6480(03)00159-X.

Gonzalvez F, Gottlieb E. 2007. Cardiolipin: setting the beat of apoptosis. Apoptosis 12:877-885. doi: 10.1007/ s10495-007-0718-8.

Gosalbes MJ, Latorre A, Lamelas A, Moya A. 2010. Genomics of intracellular symbionts in insects. International Journal of Medical Microbiology 300:271-278. doi: 10.1016/j.ijmm.2009.12.001.

Grönke S, Mildner A, Fellert S, Tennagels N, Petry S, Müller G, Jäckle H, Kühnlein RP. 2005. Brummer lipase is an evolutionary conserved fat storage regulator in Drosophila. Cell Metabolism 1:323-330. doi: 10.1016/j. cmet.2005.04.003.

Grönke S, Müller G, Hirsch J, Fellert S, Andreou A, Haase T, Jäckle H, Kühnlein RP. 2007. Dual lipolytic control of body fat storage and mobilization in Drosophila. PLOS Biology 5:e137. doi: 10.1371/journal.pbio.0050137.

Hackett KJ, Clark TB. 1979. Ecology of Spiroplasmas. In: Whitcomb RF, Tully JG, editors. The Mycoplasmas. New York: Academic Press. p. 113-200.

Haselkorn TS, Watts TD, Markow TA. 2013. Density dynamics of diverse Spiroplasma strains naturally infecting different species of Drosophila. Fly 7:204-210. doi: 10.4161/fly.25469.

Hawthorne JN, Ansell GB. 1982. Phospholipids. Elsevier Biomedical.

Hedges LM, Brownlie JC, O'Neill SL, Johnson KN. 2008. Wolbachia and virus protection in insects. Science 322:702. doi: 10.1126/science.1162418.

Herren JK, Lemaitre B. 2011. Spiroplasma and host immunity: activation of humoral immune responses increases endosymbiont load and susceptibility to certain Gram-negative bacterial pathogens in Drosophila melanogaster. Cellular Microbiology 13:1385-1396. doi: 10.1111/j.1462-5822.2011.01627.x.

Herren JK, Paredes JC, Schüpfer F, Lemaitre B. 2013. Vertical transmission of a Drosophila endosymbiont via cooption of the yolk transport and internalization machinery. mBio 4:e00532-12. doi: 10.1128/mBio.00532-12.

Hildebrandt A, Bickmeyer I, Kühnlein RP. 2011. Reliable Drosophila body fat quantification by a coupled colorimetric assay. PLOS ONE 6:e23796. doi: 10.1371/journal.pone.0023796.

Hilgenboecker K, Hammerstein P, Schlattmann P, Telschow A, Werren JH. 2008. How many species are infected with Wolbachia?-a statistical analysis of current data. FEMS Microbiology Letters 281:215-220. doi: 10.1111/j.1574-6968.2008.01110.x.

Hsu FF, Turk J. 2006. Characterization of cardiolipin from Escherichia coli by electrospray ionization with multiple stage quadrupole ion-trap mass spectrometric analysis of $[\mathrm{M}-2 \mathrm{H}+\mathrm{Na}]-$ ions. Journal of the American Society for Mass Spectrometry 17:420-429. doi: 10.1016/j.jasms.2005.11.019.

Hutchins PM, Barkley RM, Murphy RC. 2008. Separation of cellular nonpolar neutral lipids by normal-phase chromatography and analysis by electrospray ionization mass spectrometry. The Journal of Lipid Research 49:804-813. doi: 10.1194/jlr.M700521-JLR200.

Ja WW, Carvalho GB, Mak EM, de la Rosa NN, Fang AY, Liong JC, Brummel T, Benzer S. 2007. Prandiology of Drosophila and the CAFE assay. Proceedings of the National Academy of Sciences of USA 104:8253-8256. doi: 10.1073/pnas.0702726104.

Jaenike J. 2009. Coupled population dynamics of endosymbionts within and between hosts. Oikos 118:353-362. doi: 10.1111/j.1600-0706.2008.17110.x.

Jaenike J, Unckless R, Cockburn SN, Boelio LM, Perlman SJ. 2010. Adaptation via symbiosis: recent spread of a Drosophila defensive symbiont. Science 329:212-215. doi: 10.1126/science.1188235. 
Klein CC, Cottret L, Kielbassa J, Charles H, Gautier C, Ribeiro de Vasconcelos AT, Lacroix V, Sagot MF. 2012. Exploration of the core metabolism of symbiotic bacteria. BMC Genomics 13:438. doi: 10.1186/ 1471-2164-13-438.

Login FH, Balmand S, Vallier A, Vincent-Monegat C, Vigneron A, Weiss-Gayet M, Rochat D, Heddi A. 2011. Antimicrobial peptides keep insect endosymbionts under control. Science 334:362-365. doi: 10.1126/ science.1209728.

Kukasik P, van Asch M, Guo H, Ferrari J, Godfray HC. 2012. Unrelated facultative endosymbionts protect aphids against a fungal pathogen. Ecology Letters 16:214-218. doi: 10.1111/ele.12031.

Marois E, Eaton S. 2007. RNAi in the Hedgehog signaling pathway: pFRiPE, a vector for temporally and spatially controlled RNAi in Drosophila. Methods in Molecular Biology 397:115-128. doi: 10.1007/ 978-1-59745-516-9_10.

Martins AB, Ventura IM, Klaczko LB. 2010. Spiroplasma infection in Drosophila melanogaster: what is the advantage of killing males? Journal of Invertebrate Pathology 105:145-150. doi: 10.1016/j.jip.2010.06.002.

McGuire SE, Le PT, Osborn AJ, Matsumoto K, Davis RL. 2003. Spatiotemporal rescue of memory dysfunction in Drosophila. Science 302:1765-1768. doi: 10.1126/science.1089035.

Min KT, Benzer S. 1997. Wolbachia, normally a symbiont of Drosophila, can be virulent, causing degeneration and early death. Proceedings of the National Academy of Sciences of USA 94:10792-10796. doi: 10.1073/ pnas.94.20.10792.

Moran NA, McCutcheon JP, Nakabachi A. 2008. Genomics and evolution of heritable bacterial symbionts. Annual Review of Genetics 42:165-190. doi: 10.1146/annurev.genet.41.110306.130119.

Moreira LA, Iturbe-Ormaetxe I, Jeffery JA, Lu G, Pyke AT, Hedges LM, Rocha BC, Hall-Mendelin S, Day A, Riegler M, Hugo LE, Johnson KN, Kay BH, McGraw EA, van den Hurk AF, Ryan PA, O'Neill SL. 2009. A Wolbachia symbiont in Aedes aegypti limits infection with dengue, Chikungunya, and Plasmodium. Cell 139:1268-1278. doi: 10.1016/j.cell.2009.11.042.

Nishikori K, Morioka K, Kubo T, Morioka M. 2009. Age- and morph-dependent activation of the lysosomal system and Buchnera degradation in aphid endosymbiosis. Journal of Insect Physiology 55:351-357. doi: 10.1016/j.jinsphys.2009.01.001.

Oliver KM, Russell JA, Moran NA, Hunter MS. 2003. Facultative bacterial symbionts in aphids confer resistance to parasitic wasps. Proceedings of the National Academy of Sciences of USA 100:1803-1807. doi: 10.1073/ pnas.0335320100.

Palm W, Sampaio JL, Brankatschk M, Carvalho M, Mahmoud A, Shevchenko A, Eaton S. 2012. Lipoproteins in Drosophila melanogaster-assembly, function, and influence on tissue lipid composition. PLOS Genetics 8:e1002828. doi: 10.1371/journal.pgen.1002828.

Partridge L, Green A, Fowler K. 1987. Effects of egg-production and of exposure to males on female survival in Drosophila melanogaster. Journal of Insect Physiology 33:745-749. doi: 10.1016/0022-1910(87)90060-6.

Pool JE, Wong A, Aquadro CF. 2006. Finding of male-killing Spiroplasma infecting Drosophila melanogaster in Africa implies transatlantic migration of this endosymbiont. Heredity 97:27-32. doi: 10.1038/sj.hdy.6800830.

Robertson DK, Gu L, Rowe RK, Beatty WL. 2009. Inclusion biogenesis and reactivation of persistent Chlamydia trachomatis requires host cell sphingolipid biosynthesis. PLOS Pathogens 5:e1000664. doi: 10.1371/journal. ppat.1000664.

Russell CW, Bouvaine S, Newell PD, Douglas AE. 2013. Shared metabolic pathways in a coevolved insect-bacterial symbiosis. Applied and Environmental Microbiology 79:6117-6123. doi: 10.1128/AEM.01543-13.

Sakaguchi B, Poulson DF. 1961. Distribution of "sex-ratio" agent in tissues of Drosophila willistoni. Genetics 46:1665-1676.

Serbus LR, Sullivan W. 2007. A cellular basis for Wolbachia recruitment to the host germline. PLOS Pathogens 3:e190. doi: 10.1371/journal.ppat.0030190.

Sgrò CM, Partridge L. 1999. A delayed wave of death from reproduction in Drosophila. Science 286:2521-2524. doi: 10.1126/science.286.5449.2521.

Shen LR, Lai CQ, Feng X, Parnell LD, Wan JB, Wang JD, Li D, Ordovas JM, Kang JX. 2010. Drosophila lacks C20 and C22 PUFAs. The Journal of Lipid Research 51:2985-2992. doi: 10.1194/jlr.M008524.

Sieber MH, Thummel CS. 2012. Coordination of triacylglycerol and cholesterol homeostasis by DHR96 and the Drosophila LipA homolog magro. Cell Metabolism 15:122-127. doi: 10.1016/j.cmet.2011.11.011.

Soller M, Bownes M, Kubli E. 1999. Control of oocyte maturation in sexually mature Drosophila females. Developmental Biology 208:337-351. doi: 10.1006/dbio.1999.9210.

Teixeira L, Ferreira Á, Ashburner M. 2008. The bacterial symbiont Wolbachia induces resistance to RNA viral infections in Drosophila melanogaster. PLOS Biology 6:e2. doi: 10.1371/journal.pbio.1000002.

Unckless RL, Boelio LM, Herren JK, Jaenike J. 2009. Wolbachia as populations within individual insects: causes and consequences of density variation in natural populations. Proceedings of the Royal Society B: Biological Sciences 276:2805-2811. doi: 10.1098/rspb.2009.0287.

Unckless RL, Jaenike J. 2012. Maintenance of a male-killing Wolbachia in Drosophila innubila by male-killing dependent and male-killing independent mechanisms. Evolution 66:678-689. doi: 10.1111/j.1558-5646.2011.01485.x.

Vijendravarma RK, Narasimha S, Kawecki TJ. 2012. Evolution of foraging behaviour in response to chronic malnutrition in Drosophila melanogaster. Proceedings of the Royal Society B: Biological Sciences 279:3540-3546. doi: 10.1098/rspb.2012.0966.

Visser B, Le Lann C, den Blanken FJ, Harvey JA, van Alphen JJM, Ellers J. 2010. Loss of lipid synthesis as an evolutionary consequence of a parasitic lifestyle. Proceedings of the National Academy of Sciences of USA 107:8677-8682. doi: 10.1073/pnas.1001744107. 
Wernegreen JJ. 2012. Endosymbiosis. Current Biology 22:R555-R561. doi: 10.1016/j.cub.2012.06.010.

Werren JH, O'Neill SL. 1997. The evolution of heritable symbionts. In: O'Neill SL, Hoffmann AA, Werren JH, editors. Influential passengers: inherited microorganisms and arthropod reproduction. Oxford: Oxford University Press.

Xie J, Vilchez I, Mateos M. 2010. Spiroplasma bacteria enhance survival of drosophila hydei attacked by the parasitic wasp Leptopilina Heterotoma. PLOS ONE 5:e12149. doi: 10.1371/journal.pone.0012149.

Zientz E, Dandekar T, Gross R. 2004. Metabolic interdependence of obligate intracellular bacteria and their insect hosts. Microbiology and Molecular Biology Reviews 68:745-770. doi: 10.1128/MMBR.68.4.745-770.2004. 